\title{
Los Mapas Conceptuales como Estrategia que Permite Mejorar el Proceso de Enseñanza Aprendizaje de la Neuroanatomía
}

\author{
Concept Maps as a Strategy for Improving the Teaching Learning Process in Neuroanatomy
}

"Adriana Margarita Flórez-Uribe; ** Jaime Otoniel Ayala-Pimentel \& **Carlos Arturo Conde Cotes

FLÓREZ-URIBE, A. M.; AYALA-PIMENTEL, J. O. \& CONDE, C. C. A. Los mapas conceptuales como estrategia que permite mejorar el proceso de enseñanza aprendizaje de la neuroanatomía. Int. J. Morphol., 29(1):84-89, 2011.

RESUMEN: Los mapas conceptuales son una estrategia de enseñanza aprendizaje que permite organizar los contenidos de una temática determinada facilitando su interpretación, comprensión y análisis. Su construcción en forma grupal incrementa la capacidad de los estudiantes de aprender de modo significativo debido al aumento del interés y la atención para integrar los conceptos más relevantes en forma cooperativa. El objetivo de este trabajo fue evaluar el rendimiento académico de los estudiantes que cursaron la asignatura morfofisiología II en la Universidad Industrial de Santander (UIS) al implementar el uso de los mapas conceptuales como herramienta pedagógica para la enseñanza de la anatomía funcional del sistema nervioso. Se realizó un estudio de tipo prospectivo analítico con mediciones transversales en cuatro momentos en 130 estudiantes de medicina (II semestre 2006) que cursaban la asignatura morfofisiología II a quienes durante los dos primeros cortes se les transmitió la información en forma de cátedra magistral y para el tercer corte se dividieron en grupos de trabajo que utilizaron como método activo de enseñanza los mapas conceptuales. Los resultados de sus exámenes académicos escritos se compararon utilizando la prueba de Bonferroni $(\mathrm{p}<0.05)$ cuando los ANOVA indicaron diferencias estadísticamente significativas. Los resultados demostraron que las calificaciones de las evaluaciones del tercer corte fueron significativamente mejores que las de los otros, lo que nos sugiere que la realización de mapas conceptuales en forma cooperativa ofrece ventajas al estudiante entre las que se encuentra la mejoría de su rendimiento académico.

PALABRAS CLAVE: Neuroanatomía; Aprendizaje significativo; Mapa conceptual.

\section{INTRODUCCIÓN}

En la escuela de medicina de la Universidad Industrial de Santander (UIS), Colombia, Sudamérica, la enseñanza de la anatomía y la fisiología se imparte en el primer año de la carrera con las asignaturas Morfofisiología I y II. En su metodología se utilizan la clase magistral y las prácticas de laboratorio que incluyen el estudio de estructuras anatómicas en especímenes cadavéricos. La proporción de reprobados en estas materias, antes de la realización del estudio se encuentra entre un 18 al $24 \%$ del total del grupo. A pesar que la conferencia magistral ha mostrado su efectividad en la trasmisión de contenidos ya elaborados (Hudson \& Buckley, 2004), es una actividad que tiene dentro de sus debilidades el estar centrada en el profesor. Para romper este esquema se han desarrollado en algunas universidades, a nivel mundial, experiencias innovadoras de tipo participativo que han mostrado su eficacia sobre esta metodología tradicional (Gal-iglesias et al., 2009; Inzunza et al., 2007; AyalaPimentel et al., 2009).

En la UIS se ha generado una revisión crítica de los procesos de enseñanza aprendizaje de la anatomía, que han conducido al uso de herramientas activas como los mapas conceptuales, definidos como la representación visual, lógica y coherente de la estructura cognitiva de un individuo relacionada con el conocimiento de un argumento preciso (Hernández, 2007) que puede ser utilizado como una estrategia de enseñanza o un método de aprendizaje (Novak \& Gowin, 1988). Están constituidos por tres elementos básicos: los conceptos que corresponden a una palabra o una frase nominal muy breve que se emplea para designar obje-

\footnotetext{
* Grupo Neurociencias y Comportamiento, Universidad Industrial de Santander - Universidad Pontificia Bolivariana, Bucaramanga, Colombia. ***Profesor Titular Departamento Ciencias Básicas. Escuela Medicina, Universidad Industrial de Santander Colombia.
} 
tos, ideas o eventos que generalmente se colocan dentro de un círculo; las palabras de enlace que unen los conceptos y señalan los tipos de relación existente entre ambos y las proposiciones que corresponden a unidades semánticas (Ontoria, 1994; Díaz Barriga \& Hernández, 1999 ) (Fig. 1). El objetivo del presente trabajo fue evaluar la eficacia en el mejoramiento del rendimiento académico de los estudiantes que cursaron la asignatura morfofisiología II en la Universidad Industrial de Santander (UIS), al implementar el uso de los mapas conceptuales como herramienta pedagógica activa para la enseñanza de la anatomía funcional del sistema nervioso.

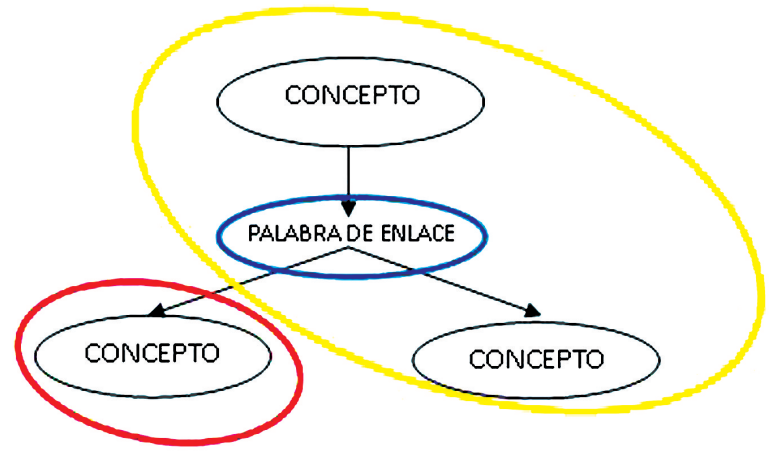

Fig. 1. Representa los componentes de un mapa conceptual en el que los elementos que constituyen una proposición se encuentran rodeados en elipses amarillo. Los conceptos o términos conceptuales se ubican en el interior de los cuadros de texto y fuera de ellos las palabras de enlace, componentes que se hallan encerrados en elipses rojas y azules respectivamente.

\section{MATERIAL Y MÉTODO}

Diseño del estudio. El presente es un estudio de tipo prospectivo analítico con mediciones transversales en cuatro momentos, fue llevado a cabo durante el segundo periodo académico del año 2006 en las secciones de morfología y fisiología de la escuela de medicina de la UIS. En él se intervino un grupo de estudiantes con la metodología de mapas conceptuales, donde los docentes tenían un nivel formativo de maestría y la intensidad horaria para el desarrollo del curso fue igual para todos los estudiantes.

Tabla I. Se evaluaron 130 estudiantes, los cuales tomaron clases teóricas como un solo grupo. Se organizaron en 4 grupos para las prácticas de laboratorios. Su distribución por género y por grupos de los estudiantes corresponde a lo mostrado en la Tabla.

\begin{tabular}{lccccc} 
& GRUPOA & GRUPOB & GRUPOC & GRUPOD & TOTAL \\
\hline HOMBRES & 13 & 17 & 16 & 18 & 64 \\
MUJERES & 23 & 23 & 9 & 11 & 66 \\
\hline
\end{tabular}

Participantes. Participaron 130 estudiantes de segundo semestre de la carrera de medicina quienes cursaban la asignatura morfofisiología II que corresponde a los tópicos de anatomía funcional de los sistemas neuroendocrino, inmunitario y reproductor masculino y femenino.

Metodología. Las actividades realizadas consistían en cinco encuentros teóricos semanales, dos de trabajo práctico en el anfiteatro y uno en el laboratorio de histología, cada uno con una duración de dos horas, durante las dieciséis semanas del curso. Las clases teóricas las recibieron como un solo colectivo y para las demás actividades, fueron divididos en 4 grupos; A, B, C y D, con 36, 40, 25 y 29 estudiantes respectivamente (Tabla I). El desarrollo de la asignatura consistió en cuatro cortes donde se realizaron evaluaciones teórico-prácticas, cada cuatro semanas, con pruebas de preguntas de selección múltiple y de identificación de estructuras macro y microscópicas referentes a los contenidos estudiados en cada corte.

Los temas abordados en los tres primeros cortes se relacionaban con la anatomía funcional del sistema nervioso, mientras que los tratados en el cuarto corte hacían referencia a los sistemas endocrino, inmunitario y reproductor.

Intervención Pedagógica. La metodología utilizada en el aula de clase, durante el primero, segundo y cuarto cortes consistió en el uso de la conferencia magistral. Para el desarrollo del tercer corte del programa se utilizó como estrategia de trabajo los mapas conceptuales, la cual se llevó a cabo en tres etapas, así:

Primera Etapa: El primer día de clases de este corte se conformaron grupos de cinco estudiantes a los que se les explicó el significado, las características, los propósitos y la forma de construir los mapas conceptuales (Novak \& Cañas, 2006). Se hizo énfasis en lo fundamental que es la consulta en textos para comprender mejor los principales conceptos del tema, reflexionar sobre sus relaciones y las que se podían establecer con las concepciones previas.

Segunda Etapa: Esta etapa tiene tres momentos. En el primero el profesor exponía el tema a tratar en forma concisa y breve y explicaba la metodología de cada clase, la cual podía variar de acuerdo a: trabajaba.

Entregar una lista de los conceptos sobre la cual se

Suministrar mapas incompletos, que en ocasiones incluían errores, para ser reelaborados.

De un texto suministrado el estudiante extraía un listado de conceptos para construir su versión de mapa conceptual. 
Un segundo momento, en el que los estudiantes reunidos por grupos leían en distintas fuentes bibliográficas, analizaban, discutían y organizaban sus ideas para realizar el trabajo propuesto. En el último momento, se socializaban los resultados en sesión plenaria, se resolvían dudas, se clarificaban ideas, y se enriquecían los trabajos con los aportes de los otros grupos. En este momento el profesor completaba la explicación con la ayuda del proyector de diapositivas e inducía a la reflexión sobre el proceso seguido.

Tercera Etapa: En esta última parte del proceso, denominada de trabajo independiente, el estudiante analizaba y evaluaba su trabajo realizado en clase y elaboraba el informe final que consistía en la entrega de cinco mapas por colectivo de trabajo.

Evaluación de la eficacia. En la evaluación de la eficacia de la adquisición de aprendizaje fue utilizado en los cuatro cortes un examen de 50 preguntas de selección múltiple, de única respuesta, con 4 distractores obtenidas de un banco de preguntas previo, elaborado de manera independiente a la formulación del protocolo de este estudio; para aprobar el examen los estudiantes debían contestar acertadamente 30 preguntas.

Análisis. El rendimiento académico se evaluó mediante las calificaciones teóricas de las evaluaciones de cada corte. Se realizaron cálculos de estadística descriptiva para la calificación de cada corte (promedio, desviación y error estándar, distribuciones de frecuencias absolutas, relativas y acumu- ladas) discriminada por grupos y para el total de estudiantes. Las comparaciones entre las calificaciones de cada corte se realizaron mediante ANOVA para medidas repetidas seguidas de pruebas de Bonferroni para comparaciones múltiples corregidas cuando el ANOVA mostró diferencias significativas. Los cálculos estadísticos se realizaron utilizando Excel y Sigmastat 2.5.

\section{RESULTADOS}

Se evaluó el desempeño académico de los grupos frente a cada previo teórico cuyos promedios y desviaciones estándar se incluyen en la Tabla II. En ella se muestra que la evaluación teórica con mejores resultados fue la correspondiente al tercer corte en forma global y estratificada por grupos, con un mejor desempeño en las cuatro evaluaciones para el grupo B, aunque en el corte en que se realizó la intervención los resultados de todos los grupos fueron homogéneos.

Un ANOVA de medidas repetidas para la comparación de los 4 cortes, que incluyen todos los estudiantes, mostró diferencias significativas (F 3,387: 39.116, p<0,001) donde las comparaciones múltiples con Bonferroni indicaron que: Las calificaciones del tercer parcial fueron significativamente mayores que las de los otros parciales. La del parcial 4 fue mayor que las de 1 y 2 y la del primero fue mayor que la del segundo (Tabla III y Fig. 2).

Tabla II. En la tabla se observa el promedio \pm 1 desviación estándar de la media de los parciales teóricos por grupos. PTn= Parcial teórico "n".

\begin{tabular}{|c|c|c|c|c|c|}
\hline GRUPO & $\mathrm{N}$ & PT1 & PT2 & PT3 & PT4 \\
\hline A & 36 & $2,656(0,558)$ & $2,622(0,539)$ & $3,30310,58$ & $2,875(0,835)$ \\
\hline B & 40 & $3,112(0,516)$ & $2,867(0,592)$ & $3,502(0,554)$ & $3,407 \quad(0,59)$ \\
\hline c & 25 & $2,776(0,551)$ & $2,44 \quad(0,668)$ & $3,22 \quad(0,603)$ & $3,184(0,602)$ \\
\hline D & 29 & $3,052(0,537)$ & $2,928(0,507)$ & $3,234(0,635)$ & $2,821(0,931)$ \\
\hline TOTAL & 130 & $2,908(0,568)$ & $2,731(0,598)$ & $3,333(0,596)$ & $3,086(0,782)$ \\
\hline
\end{tabular}

Tabla III. Resultados obtenidos con la prueba de Bonferroni con las comparaciones entre los parciales teóricos (PT1,2,3 y 4) para cada grupo (A, B, C y D) e incluyendo todos los participantes.

\begin{tabular}{cccc}
\hline Comparaciones & Dif medias & $\mathbf{t}$ & $\mathbf{P}$ \\
\hline PT3 vs. PT2 & 0,602 & 10,354 & $<0,001$ \\
PT3 vs. PT1 & 0,425 & 7,313 & $<0,001$ \\
PT3 Vs. PT4 & 0,247 & 4,254 & $<0,001$ \\
PT4 vs. PT2 & 0,355 & 6,109 & $<0,001$ \\
PT4 vs. PT1 & 0,178 & 3,068 & 0,014 \\
PT1 vs. PT2 & 0,177 & 3,041 & 0,015 \\
\hline
\end{tabular}




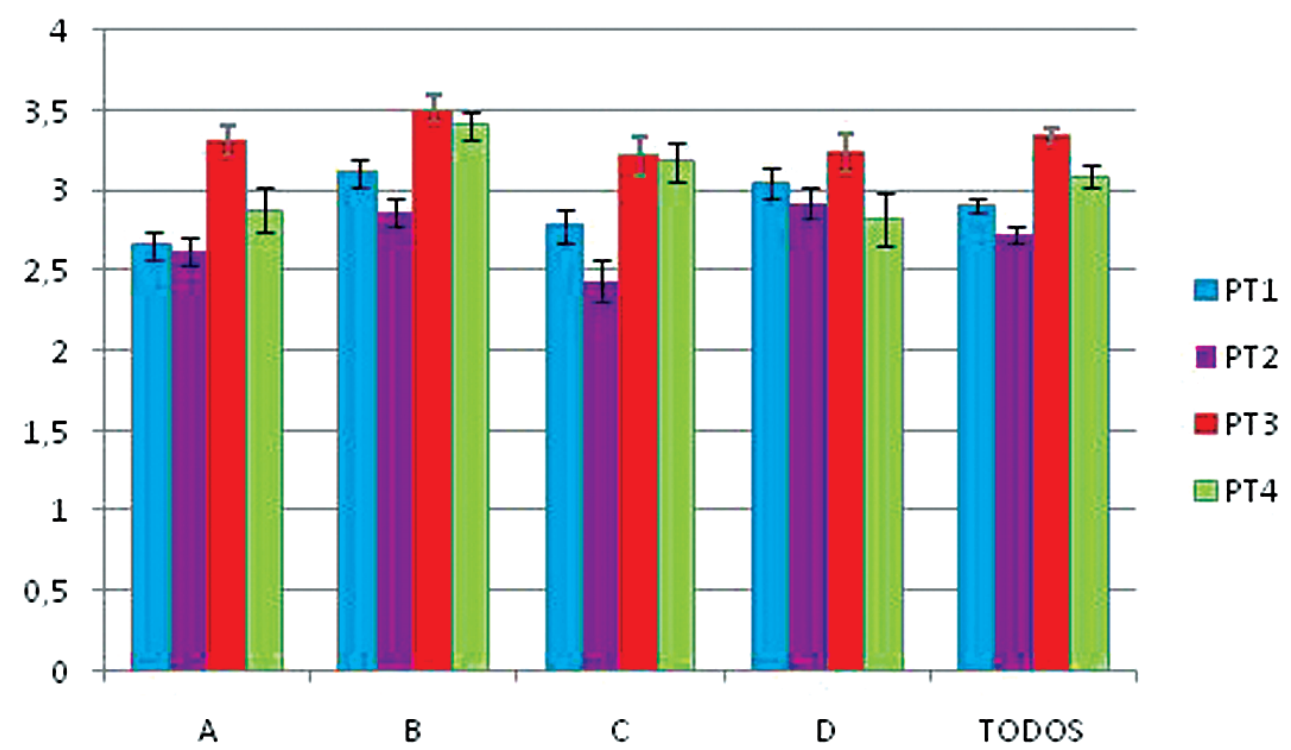

Fig. 2. Representa los promedios y errores estándar del promedio de los parciales. Para identificar las diferencias significativas ver el texto.

\section{Frecuencias Absolutas de los Parciales Teoricos}

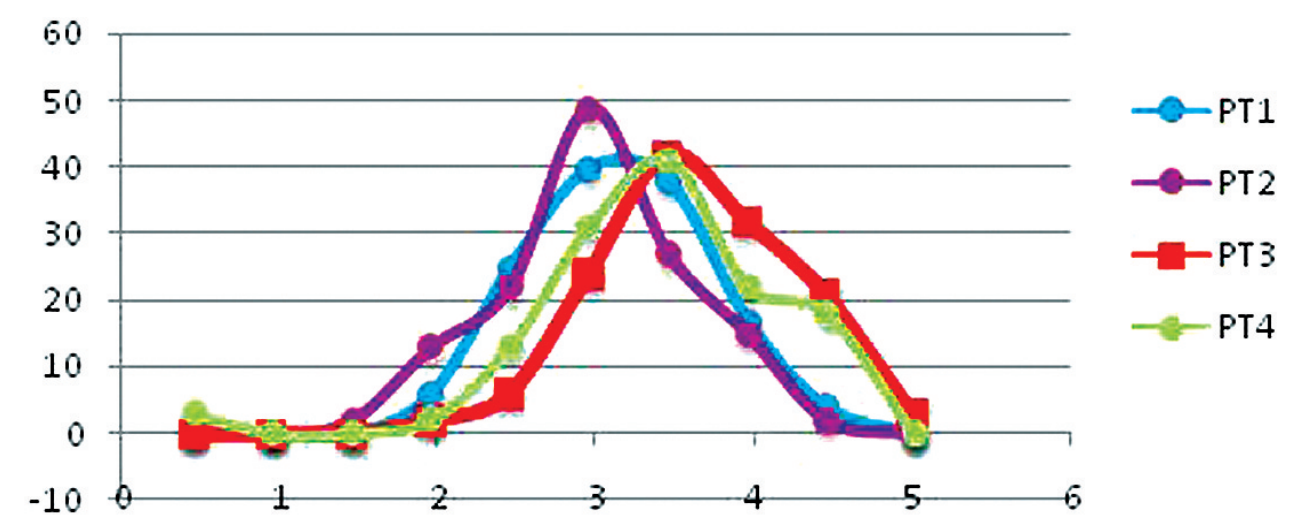

Fig. 3. Frecuencias absolutas de los cuatro parciales teóricos. Las calificaciones van de 0 a 5 , siendo 5 la máxima nota posible a obtener. Las líneas de colores correspondes a las frecuencias absolutas en cada uno de los cuatro parciales teóricos, azul, morado, rojo y verde respectivamente.
Las frecuencias absolutas para cada corte indican que la mejoría académica en la tercera evaluación fue significativa respecto a las demás (Fig. 3). En la tabla IV se observa el comportamiento de los estudiantes a través de los parciales por rangos de notas, en ella podemos evidenciar que la población por debajo de 2,95 en el tercer corte correspondía al 24,64\% de los estudiantes mientras que para el primero, segundo y cuarto cortes equivalían a un $54,62 \%, \quad 66,15 \%$ y $37,69 \%$ respectivamente, lo que indica un mejor desempeño grupal al reducirse el porcentaje de personas que no aprobaron la evaluación teórica correspondiente al tercer corte. De igual manera se observó en éste corte que el 2,31\% de los estudiantes obtuvieron notas superiores a 4,45 mientras que en las demás evaluaciones no existió ningún estudiante en este rango.

Tabla IV. Porcentaje de rangos acumulados de los cuatro cortes. Se evidencia la diferencia porcentual correspondiente al rango de 2,95 que marca el punto de aprobación de la evaluación teórica.

\begin{tabular}{|c|c|c|c|c|c|c|c|c|}
\hline \multirow[t]{2}{*}{ Rango } & \multicolumn{2}{|c|}{ PT1 } & \multicolumn{2}{|r|}{ PT2 } & \multicolumn{2}{|c|}{ PT3 } & \multicolumn{2}{|r|}{ PT4 } \\
\hline & Frecuencia & $\%$ Acumulado & Frecuencia & $\%$ Acumulado & Frecuencia & $\%$ Acumulado & Frecuencia & \% Acumulado \\
\hline 0,45 & 0 & 0,00 & 0 & 0,00 & 0 & 0,00 & 3 & 2,31 \\
\hline 0,95 & 0 & 0,00 & 0 & 0,00 & 0 & 0,00 & 0 & 2,31 \\
\hline 1,45 & 0 & 0,00 & 2 & 1,54 & 0 & 0,00 & 0 & 2,31 \\
\hline 1,95 & 6 & 4,62 & 13 & 11,54 & 2 & 1,54 & 2 & 3,85 \\
\hline 2,45 & 25 & 23,85 & 22 & 28,46 & 6 & 6,15 & 13 & 13,85 \\
\hline 2,95 & 40 & 54,62 & 49 & 66,15 & 24 & 24,62 & 31 & 37,69 \\
\hline 3,45 & 38 & 83,85 & 27 & 86,92 & 42 & 56,92 & 41 & 69,23 \\
\hline 3,95 & 17 & 96,92 & 15 & 98,46 & 32 & 81,54 & 22 & 86,15 \\
\hline 4,45 & 4 & 100 & 2 & 100 & 21 & 97,69 & 18 & 100 \\
\hline 5 & 0 & 100 & 0 & 100 & 3 & 100 & 0 & 100 \\
\hline
\end{tabular}




\section{DISCUSIÓN}

Teniendo como premisa el logro del aprendizaje significativo con menor esfuerzo y la generación de conocimiento con la mejoría del rendimiento académico, por parte del estudiante, se diseñó y aplicó un nuevo proceso didáctico de construcción de conocimiento por descubrimiento basado en las teorías cognitivas de aprendizaje de Vigotsky (1979, 1985), Pozo (2003) y, principalmente del aprendizaje significativo de David Ausubel (Ausubel et al., 1996), que involucraba el uso de mapas conceptuales. Esta estrategia innovadora para la enseñanza de la anatomía del sistema nervioso, ha sido utilizada en diversos escenarios pedagógicos dentro y fuera del área de la salud con resultados satisfactorios.

El presente trabajo nos permite acercarnos a establecer comparaciones entre las dos formas de instrucción aplicadas, que conducen a la consolidación del aprendizaje significativo por recepción o por descubrimiento. Los resultados de la evaluación escrita de conocimientos nos muestra un mejor promedio grupal en el corte en que se manejó como instrucción escolar los mapas conceptuales (Fig. 2). De igual manera, al analizar la escala de notas durante la tercera prueba se observa como un mayor número de alumnos se localizan en el intervalo más alto, mientras que un número menor de ellos se ubican en el rango inferior de las notas (Tabla IV y Fig. 3).

Existen diversas estrategias utilizadas para la enseñanza de la anatomía del sistema nervioso, basadas en el constructivismo. En la universidad del estado de Youngstown, en Ohio, se aplicó una técnica pedagógica que consistía en el uso de un tablero magnético y un kit con imanes que representan estructuras y componentes del sistema nervioso (Spine Board), donde se comparó el grupo de la estrategia con un grupo que repasaba los apuntes de clase, sin encontrarse diferencias significativas $(\mathrm{p}=0,061)$ al evaluar su rendimiento académico (Krontiris-Litowitz, 2008). De igual forma la universidad de Massachussets evaluó y comprobó que la utilización del repaso es un método útil para mejorar la retención de los conceptos en neuroanatomía $(\mathrm{p}=0,000)$ (Billings-Gagliardi \& Mazor, 2009).

La metodología utilizada en nuestro estudio nos permite, al igual que estudio de la universidad de Massachussets, visualizar que existe una mejoría del rendimiento académico, en el que el error "es un indicador o sensor de procesos que no han funcionado como esperábamos" (De La Torre \& Barrios, 2000) que pueden ser corregidos en forma cooperativa, donde el alumno es un procesador activo de la información con la que genera conocimientos, desarrolla sus capacidades y transforma su realidad.

En el ámbito pedagógico el desarrollo de los objetivos de una disciplina pueden requerir del uso complementario de un amplio repertorio de estrategias (Joyce \& Weil, 1985; Pozo \& Gómez Crespo, 1998). Con nuestro trabajo, presentamos una estrategia de enseñanza aprendizaje, probada, que permitirá a los docentes en el área de la neuroanatomía implementarla de acuerdo al estilo de aprendizaje predominante en sus estudiantes, su experiencia y los objetivos propuestos en el desarrollo de su asignatura.

FLÓREZ-URIBE, A. M.; AYALA-PIMENTEL, J. O. \& CONDE, C. C. A. Concept maps as a strategy for improving the teaching learning process in neuroanatomy. Int. J. Morphol., 29(1):84-89, 2011.

SUMMARY: The present study describes the implementation of conceptual maps as a pedagogical strategy that tends to transform the passive students into an active producer. The use of concept maps in groups encourages students to meaningful learning, increasing interest and attention in relevant concepts working in a cooperative way. The objective was to measure the improvement of academic performance of the student when implementing the strategy of conceptual maps as a pedagogical tool of students of morphophysiology II at the Universidad Industrial de Santander (UIS) for teaching the functional anatomy of the nervous system. An analytic prospective with four transversal sections study was conducted in 130 students of medicine (II semesters of 2006) Bucaramanga, Santander at 2006, who attended the course morphophysiology II. For the first and the second section the students received master classes and for the third section the group was divided and the active method of concept maps was applied. The multiple corrected comparisons were made using the Bonferroni test $(\mathrm{p}<0.05)$ when the ANOVA shows significant statistical differences. All student received master classes as a group. For other activities they were divided in 4 groups; A, B, C and D with 36, 40, 25 and 29 students respectively. It is important that at the third period the performance of the group was homogeneous, finding not difference between groups. An ANOVA for all students in comparison of the 4 periods showed significant differences $(F 3,387$ : 39,116, $\mathrm{p}<0,001)$ where the comparison with Bonferroni demonstrated that performance in the third period was significantly better than in the other periods. Using concept maps as an strategy for actively constructing knowledge by students confirms that the process of learning is active and was reflected in the improvement of their performance.

KEY WORDS: Neuroanatomy; Critical learning; Concept maps. 


\section{REFERENCIAS BIBLIOGRÁFICAS}

Ausubel, D.; Novak, J. \& Hanesian, H. Psicología educativa: Un punto de vista cognoscitivo. 9. ed. México, Trillas, 1996.

Ayala-Pimentel, J. O.; Diaz-Perez J. A. \& Orozco-Vargas, L. C. Eficacia de la utilización de estilos de aprendizaje en conjunto con mapas conceptuales y aprendizaje basado en la resolución de problemas para el aprendizaje de neuroanatomía. Educ. Méd., 12:25-31, 2009.

Billings-Gagliardi, S. \& Mazor, K. Effects of review on medical student's recall of differente types of neuroanatomical content. Acad. Med., 84:S34-7, 2009.

De la Torre, S. \& Barrios, O. Estrategias Didáctica Innovadoras. El error como estrategia didáctica. Barcelona, Ediciones Octaedro, 2000.

Díaz Barriga, F. \& Hernández, G. Estrategias docentes para un aprendizaje significativo. Una interpretación constructivista. México, Mcgraw-Hill, 1999.

Gal-Iglesias, B.; Busturia-Berrade, I. \& Garrido-Astray, M. C. Nuevas metodologías docentes aplicadas al estudio de la fisiología y la anatomía: estudio comparativo con el método tradicional. Educ. Méd., 12:117-24, 2009.

Hernández, F. V. Mapas conceptuales. La gestión del conocimiento en la didáctica. $2^{\mathrm{a}}$ Ed. México, Alfaomega, 2007.

Hudson, J. N. \& Buckley, P. An evaluation of case-based teaching: evidence for continuing benefit and realization of aims. Adv. Physiol. Educ., 28:15-22, 2004.

Inzunza, O.; Vargas, A. \& Bravo, H. Anatomía y Neuroanatomía, Disciplinas Perjudicadas por la Reforma Curricular. Int. J. Morphol., 25:825-30, 2007.

Joyce, B. \& Weil, M. Modelos de enseñanza. Madrid, Anaya, 1985.

Krontiris-Litowitz, J. Using truncated lectures, conceptual exercises, and manipulatives to improve learning in the neuroanatomy classroom. Adv. Physiol. Educ., 32:1526, 2008.

Novak J. \& Gowin, D. Aprendiendo a aprender. Barcelona, Ediciones Martinez Roca, 1988.
Novak. J. \& Cañas, A. The theory underlying concept maps and how to construct and use them. Technical Report IHMC CmapTools, Rev 01, 2006. Disponible en: http:// cmap.ihmc.us/publications/researchpapers/theorycmaps/ theoryunderlyingconceptmaps.htm

Ontoria A. Mapas conceptuales una técnica para aprender. Madrid, Narcea, 1994.

Pozo, J. I. Teorías cognitivas del aprendizaje. $8^{\mathrm{a}}$ Ed. Madrid, Morata, 2003.

Pozo, J. I. \& Gómez Crespo, M. A. Aprender y enseñar ciencia. Madrid, Morata, 1988.

Vigotsky, L. El desarrollo de los procesos psicológicos superiores. Barcelona, Grijalbo, 1979.

Vigotsky, L. Pensamiento y Lenguaje. Buenos Aires, Pléyade, 1985.

Dirección para correspondencia:

Jaime O. Ayala-Pimentel.

Departamento de Ciencias Básicas

Unidad de Neuroanatomía

Facultad de Salud

Universidad Industrial de Santander.

Cra 32 No 29-31

Bucaramanga

COLOMBIA

Email: jayala@uis.edu.co

Recibido : 02-09-2010

Aceptado:18-12-2010 